\title{
Narrative review of management of thyroid surgery complications
}

\author{
Shan Jin ${ }^{1,2}$, Iwao Sugitani ${ }^{2}$ \\ ${ }^{1}$ Department of General Surgery, Affiliated Hospital of Inner Mongolia Medical University, Hohhot, China; ${ }^{2}$ Department of Endocrine Surgery, \\ Nippon Medical School Graduate School of Medicine, Tokyo, Japan \\ Contributions: (I) Conception and design: Both authors; (II) Administrative support: Both authors; (III) Provision of study materials or patients: S Jin; \\ (IV) Collection and assembly of data: S Jin; (V) Data analysis and interpretation: Both authors; (VI) Manuscript writing: Both authors; (VII) Final \\ approval of manuscript: Both authors. \\ Correspondence to: Shan Jin. Department of General Surgery, Affiliated Hospital of Inner Mongolia Medical University, Tongdao North Rd 1, Hohhot \\ 010050, China. Email: jinshangood@163.com.
}

\begin{abstract}
Currently, thyroid surgery is the most common and safe operation worldwide. However, thyroidectomy is still not free from the risks of complications and death due to the anatomical structure and physiological function particularity of the thyroid gland. Postoperative complications affect the life quality and life safety of patients after surgery. The common complications include hypoparathyroidism (HP), recurrent laryngeal nerve (RLN) injury, injury to the external branch of the superior laryngeal nerve (EBSLN), postoperative bleeding (PB), thoracic duct injury, laryngeal edema, tracheospasm, tracheal injury, and esophageal injury. A severe complication, such as dyspnea, asphyxia, or thyroid crisis, might cause the death of the patient. Therefore, every thyroid surgeon's responsibility is to remain alert and aware of the occurrence of various intraoperative and postoperative complications and exercise effective prevention and treatment. This is closely related to the advancement in thyroid disease research, the increase in local anatomy knowledge, the standardization of surgical approaches, the improvement in operating skills, the application of new technologies, and the emphasis on specialty training. In addition, many complications that effect patients are much better tolerated if the patient has appropriate expectations of what the complications are and how to treat them. Open communication between surgeon and patient optimizes the potential negative effects that complications may have on patients' quality of life. This paper discusses the prevention, recognition and therapy of intraoperative and postoperative complications in thyroid surgery.
\end{abstract}

Keywords: Thyroid surgery; complication; management

Submitted Nov 29, 2020. Accepted for publication Jan 27, 2021.

doi: $10.21037 /$ gs-20-859

View this article at: http://dx.doi.org/10.21037/gs-20-859

\section{Introduction}

In 1791, Dr. Pierre Joseph Desault successfully performed the first partial thyroidectomy (1). However, the mortality associated with thyroid operations was high, and hence, thyroid surgery was banned at one point in history. In 1849, Dr. Nikolai Piringoff performed a successful thyroidectomy using ether anesthesia (1), which once again ushered in an era of thyroid surgery. Subsequently, with doctors' efforts, including Theodor Billroth, Theodor Kocher, and William Halsted, thyroid surgery developed rapidly. Especially, the surgery-related death rate declined to $<0.5 \%$ due to the contributions of Dr. Theodor Kocher (2).

Currently, thyroid surgery is the most common and safe operation worldwide and is closely related to the advancement in thyroid disease researches, the increase

^ ORCID: 0000-0001-6935-0162. 
in local anatomy knowledge, the standardization of surgical approaches, the improvement in operating skills, the application of new technologies, and the emphasis on specialty training (3-5). Nevertheless, despite that the techniques of thyroid surgery have been improving continually, thyroidectomy is still not free from the risks of complications and death due to the complexity of the anatomical structure and physiological function of the thyroid gland. In thyroid surgery, the overall incidence of complications is approximately $0-54.4 \%$ (6-9).

According to a previous meta-analysis, no significant difference was detected between the overall complication rate from traditional open surgery and that from minimally invasive (video-assisted) surgery (10). The common complications include hypoparathyroidism (HP), recurrent laryngeal nerve (RLN) injury, injury to the external branch of the superior laryngeal nerve (EBSLN), postoperative bleeding (PB), thoracic duct injury, laryngeal edema, tracheospasm, tracheal injury, and esophageal injury. A severe complication, such as dyspnea, asphyxia, or thyroid crisis, might cause the death of the patient. Therefore, vigilance against and understanding the various intraoperative and postoperative complications are mandatory skills for every thyroid surgeon in addition to preventing and treating these complications. The purpose of this study is that the various intraoperative and postoperative complications should be prevented, recognized and treated to enhance the safety profile of the thyroidectomy. We present the following article in accordance with the Narrative Review reporting checklist (available at http://dx.doi.org/10.21037/gs-20-859).

\section{Methods}

\section{Search criteria}

This review has been conducted employing the PubMed, Medline, Web of Science, and Science Direct database. Articles between January 2005 and June 2020 were searched, using the key terms "thyroidectomy", "complication", "hypoparathyroidism", "hypocalcemia", "recurrent laryngeal nerve injury", "superior laryngeal nerve injury", "postoperative bleeding", "lymphatic leakage", "chylous leakage", "tracheal collapse", "tracheomalacia", "laryngeal edema", "tracheospasm", "tracheal injury", "esophageal injury", "incision scar formation", and "skin sinus formation". Specifically, the research has been restricted using variable combinations of the keywords. As inclusion criteria, the articles had to provide data on causes of postthyroidectomy complications, management or anatomy information. Some of the articles were excluded due to irrelevance to the topic in question.

\section{Analyses}

Data were extracted from studies satisfying the inclusion and exclusion criteria. Our outcome of interest included post-thyroidectomy complication rates, complication causes, intraoperative management and postoperative treatment.

\section{Results and discussion}

\section{HP}

Hypocalcemia caused by parathyroid gland damage is a common postoperative complication. The incidence of transient hypocalcemia is approximately $1.2-40 \%$, while the incidence of permanent hypocalcemia is approximately $3 \%$ (11). The risk of postoperative hypocalcemia is related to gender (female), Grave's disease, lymph node dissection, surgical approach (total thyroidectomy, reoperation, or extensive surgery), and bleeding $(11,12)$. However, the correct identification and protection of the parathyroid glands during surgery are important prerequisites for preventing hypocalcemia $(12,13)$.

The first step to prevent injury is to accurately identify the parathyroid glands. Occasionally, it is difficult to distinguish these glands from fat granules and lymph nodes. Human parathyroid glands are round structures or flat ovoid, with an average size of approximately $6 \mathrm{~mm}$, and usually yellowish-brown. On the surface of each gland, small blood vessels are observed, which are prominent under a magnifying glass (14). Fat granules are spherical or elliptical in shape, with different sizes, and usually goldenyellow. Also, no blood vessel pattern is visible on the surface, and a fat granule can easily float in normal saline. Interestingly, the lymph nodes are pea-shaped or round, with different sizes, and pink. In a normal lymph node, a hilum can be observed, while metastatic lymph nodes feel hard to the touch. Based on these characteristics, fat granules, lymph nodes, and parathyroid glands should be readily distinguishable. Alternatively, parathyroid glands can be identified using autofluorescence and indocyanine green (ICG) fluorescence imaging techniques $(15,16)$. Any difficulty in identification might prompt surgery for rapid pathological examination, and some tissues may be cut off 
in surgery. Based on the identification of the parathyroid glands, protecting the blood supply is imperative. In order to protect the blood supply of the superior and inferior parathyroid glands, the following measures should be taken: (I) when handling the superior thyroid pole, ossify the blood vessels, keep close to the superior pole capsule, and then ligate and cut the blood vessels separately to protect the posterior branch of the superior artery; (II) when handling the lateral side of the thyroid, protect the ascending and descending branches of the inferior artery and avoid ligating the stem of the inferior artery; (III) when the blood supply of the inferior parathyroid gland originates from the lowest artery, preserve the integrity of the lowest artery; (IV) in addition to the arterial blood supply, the returning veins should also be protected; (V) maintain the integrity of adipose capsules outside the parathyroid glands to the extent practically possible; (VI) when handling blood vessels close to the parathyroid glands, conventional ligation should be selected as energy devices may cause thermal injury. During surgery, the identification and protection of the blood supply of the inferior parathyroid gland are more difficult than those of the superior parathyroid gland. Especially during central lymph node dissection, the protection of the inferior parathyroid gland is challenging. Thus, the inferior parathyroid gland may be removed for autologous transplantation. Before the end of the surgery, the blood supply status of the parathyroid gland preserved in situ should be checked carefully (17). If the color of the gland has not changed or is slightly darker than before, and the capsule is shiny, it indicates normal blood supply. If the color turns pale, it indicates ischemia, and autologous transplantation may be needed. On the other hand, if the color turns dark brown, it indicates obstruction of venous return. A fine needle may be used to puncture the gland a few times so that the dark red blood flows out. Subsequently, if the color improves, the parathyroid gland may be preserved in situ. Furthermore, if the color turns black, it indicates severe venous return obstruction. After treatment, such as puncturing and capsular incision, if the color does not improve, autologous transplantation is preferred (18). The autologous parathyroid transplantation includes tissue embedding and homogenate injection. Typically, healthy sternocleidomastoid muscles or forearm muscles are selected for transplantation. For thyroid cancer surgery, cutting off some parathyroid tissues for intraoperative rapid pathological examination before transplantation is recommended. According to studies by Sitges-Serra et al., autologous parathyroid transplantation does not improve the occurrence of postoperative permanent hypocalcemia and/or HP, emphasizing the importance of in situ parathyroid preservation $(19,20)$. Auto-fluorescence may aid parathyroid detection, but methylene blue fluorescence is needed to demonstrate viability $(21,22)$. This detection may help preserve these fragile structures and their vascularization and lower the rate of postoperative hypocalcemia (15). However, infrared autofluorescence's ability to confirm the presence of parathyroid tissue within surgical specimens was high, its power to find parathyroid glands in situ before visual recognition by surgeons was low, as described by Kose et al. (23).

In the case of acute hypocalcemia (serum calcium $<1.75 \mathrm{mmol} / \mathrm{L}$ ), even if there are no clinical symptoms, intravenous calcium supplementation is required for preventing the occurrence of serious symptoms (laryngospasm, and respiratory muscle paralysis, etc) (24). For chronic hypocalcemia, calcium and active vitamin D (calcitriol) are administered orally (11). Strikingly, patients reporting poor effects of calcium and vitamin $\mathrm{D}$ supplementation may be treated with parathyroid hormone (PTH)-related peptide replacement therapy (25). In the study by Shah et al., Teriparatide (recombinant human PTH $1-34)$ therapy $(20 \mu \mathrm{g}$ twice daily subcutaneously for 1 week) in patients with post-thyroidectomy HP was safe, rapidly eliminated hypocalcemic symptoms (26). In addition, The THYPOS Trial also confirmed that Teriparatide might prevent postsurgical hypocalcemia, shorten the duration of hospitalization, and reduce the need for calcium and vitamin D supplementation in high-risk subjects after thyroid surgery (27).

\section{RLN injury}

RLN injury is a common complication. The incidence of transient RLN palsy is $5-8 \%$, while that of permanent RLN palsy is $0.3-3 \%$ (28). Several factors may cause injury to the RLN, such as excessive traction/stretching, contusion, clamping, ligation, suturing, burning, and cutting (29). The injury may occur at any position of the RLN cervical segment. It is primarily detected within $2 \mathrm{~cm}$ before entry to the larynx, where the RLN is located at a fixed position and is closely related to Zuckerkandl's tubercle and Berry's ligament, according to the anatomy (30).

During surgery, it is easy to locate and expose the RLN at the entry to the larynx, at the junction of the inferior thyroid artery and the RLN, and in the tracheoesophageal groove at the inferior thyroid pole (30). Although RLN is 
fixedly located at the point to enter the larynx, it is prone to injury during dissection. However, at the junction of the inferior thyroid artery and the RLN, the nerve can be exposed easily, but bleeding may be caused in the small branches of the inferior artery. Also, RLN has great mobility in the tracheoesophageal groove at the inferior thyroid pole, expanding its anatomical range. A surgeon may select an appropriate approach based on his/her operating skills, clinical experience, and understanding of anatomy. In addition, learning about the anatomical structure of RLN is critical. In most cases, RLN approaches the larynx as a single trunk and branches off into an anterior and a posterior branch, but in some cases (approximately $10 \%$ ), the RLN has branches (often two and sometimes even three) before entering the larynx (31). Moreover, originating from the trunk would facilitate many small branches distributed in the esophagus and the trachea. Upon entry into the larynx, when the RLN is exposed during dissection, these branches can be injured easily, giving rise to corresponding symptoms. Additionally, a huge goiter pushes the RLN outward. The nerve often courses along the goiter's posterolateral surface instead of the dorsal side of the thyroid, thereby increasing the probability of injury. When a cancer focus infiltrates RLN, if only the nerve capsule is invaded, the nerve function is protected during surgery; however, the dissection, traction/stretching, and freeing could damage the nerve fibers. The rapid expansion of treatment methods such as dehydrated ethanol injection and radiofrequency ablation coupled with nonstandardized surgery has led to an increase in the number of reoperations. Unlike initial surgery, the surgical sites of reoperations have severe adhesions, which increases the probability of RLN injury (32). Furthermore, the surgeons should be wary of the appearance of a non-recurrent laryngeal nerve (NRLN), mainly on the right side, with an incidence rate of approximately $0.3-1.6 \%(29,33)$. Although energy devices, such as an electrosurgical knife, bipolar electrocoagulation, ultrasonic knife, and LigaSure exhibit satisfactory hemostatic effects, the improper application increases the possibility of nerve burns, scalds, as well as miscuts (34).

The routine exposure of RLN during surgery is a key factor in preventing nerve injury. During dissection, RLN exposure is dependent on the following conditions. Firstly, the surgeon is familiar with the course and anatomical structure of RLN. Secondly, the surgeon is skilled in dissecting and separating the living tissues. Lastly, the surgeon has clinical experience in dealing with various risks.
The application of intraoperative neuromonitoring (IONM) is helpful for the identification of RLN. However, IONM and conventional visual exposure do not exert a significant effect on postoperative RLN palsy, as described by Pisanu et al. $(35,36)$. IONM is essential for complex thyroidectomy or reoperations. In the event of an abnormal situation during surgery, the experience of the surgeon is significantly related to neuroprotection. Professionally trained doctors with abundant clinical experience can effectively reduce the probability of nerve injury (5). For example, during the resection of a huge goiter, the position of RLN may change; if an ultrasound or CT scan indicates the abnormal development of the right subclavian artery, an NRLN may be present on the right side; when cancer infiltrates the RLN, the surgeon knows how to perform sharp dissection to protect the nerve fibers; the surgeon is familiar with the safety risks of using energy devices and knows when to choose traditional suturing and/or knotting.

When RLN injury is confirmed, first, the type of injury should be determined. If it is nervous function-related palsy caused by ligation, suturing, and adhesion, an exploratory operation should be performed at the earliest. Importantly, nerve decompression surgery performed within 3 months can provide a satisfactory outcome (37). For patients whose nerves have been severed or cut off due to tumors, nerve repair operations, including end-to-end RLN anastomosis, free nerve grafting, and ansa cervicalis-to-recurrent laryngeal nerve (ansa-RLN) anastomosis, are required (38-40). End-to-end RLN anastomosis is easy to perform, but RLN contains contractile and diastolic fibers. The misalignment in the end-to-end anastomosis could cause paradoxical vocal fold movement. Therefore, this method is suitable for those whose nerves have not been severed completely, such that the nerve fibers at both ends are not easily misaligned during anastomosis (38). Because the trunk and the thick branches of the ansa cervicalis consist of abundant motor fibers, ansa-RLN anastomosis was used to repair the RLN; as a result, the vocal cords of most of the patients can move sufficiently, and the voices can return to normal or close to normal (40).

\section{EBSLN injury}

Reportedly, the incidence of injury to EBSLN is as high as $58 \%$ (41), which might be related to various underlying criteria (42). The anatomical correlation between EBSLN and blood vessels of the superior thyroid pole (Cernea classification) can be categorized into three types: (I) the 
EBSLN crosses the superior thyroidal vessels at least $1 \mathrm{~cm}$ above a plane horizontal to the upper edge of the superior thyroid pole (type I); (II) the distance is $<1 \mathrm{~cm}$ and is not below the plane (type II a); (III) the EBSLN is below the plane (type II b) (43). During handling of the superior pole vessels, type I poses the lowest risk of injury, while type II b poses the highest risk of injury. After crossing the superior thyroidal vessels, the EBSLN penetrates the inferior pharyngeal constrictor muscle from the medial side of the superior thyroid pole or courses along its surface, before reaching the cricothyroid muscle. The nerve fibers of the external branch are small, with a diameter of approximately $0.8 \mathrm{~mm}$, and hence, can be easily injured during dissection and freeing (42). Except in locally advanced cases, the incidence of tumor invasion into the EBSLN is low. In reoperation cases, the nerve can be damaged easily due to surgical site adhesion and unclear anatomical structure. The key to preventing injury to the EBSLN is to expose the surgical site around the superior thyroid pole. When this is not possible, the surgical incision can be extended, and when handling the blood vessels of the superior thyroid pole, the incision is kept as close to the superior pole capsule as possible to prevent damage to the type I or type II external branch. Moreover, if necessary, the sternothyroid muscle is cut off, and the blood vessels of the superior thyroid pole are ossified such that damage to the type II b external branch can be prevented. Subsequently, IONM is used to observe cricothyroid muscle tremors or electromyography (EMG) signals and accurately grasp the approximate positions of nerve fibers coursing along the surface of the muscle or within the muscle, which prevents damage to the external branch (44). In the case of unilateral EBSLN damage, neurotrophic and hormonal drugs are administered. Due to compensation by the healthy side, the symptoms of most patients improve to varying degrees within 3 months. When EBSLN damage is bilateral, the patient experiences cricothyroid muscle and inferior pharyngeal constrictor muscle dysfunction. Also, dysphagia, bucking, and dysphonia is observed, thereby necessitating surgical treatment.

\section{$P B$}

Bleeding is a severe complication that occurs within $24-48 \mathrm{~h}$ post-surgery, with an incidence of approximately $0-4.2 \%$ (45). According to a meta-analysis of 25 studies (424,563 patients) by Liu et al., bleeding after thyroid surgery is associated with older age, male gender, Graves' disease, anticoagulants, bilateral thyroid surgery, cervical lymph node dissection, and thyroid reoperation (46). In the early stage of bleeding, if the drainage is unobstructed, a large amount of fresh blood can be seen in the drain, or blood oozing from the incision is increased. When the amount of blood loss reaches a certain level, fresh blood in the drain begins to coagulate and accumulate in the surgical cavity. Patients should be aware that pain usually is a pre-emptive symptom before significant neck swelling. The patient feels difficulty in breathing when the blood accumulates to the point that the skin flaps swell. The degree of difficulty in breathing is related to not only the amount of blood loss but also the bleeding speed (47). In the case of acute arterial bleeding, the patient has difficulty in breathing and may even suffocate or die. Following surgery, the bleeding may be triggered by sneezing, coughing, emotional agitation, and intense neck movements, or even without any cause. In addition to systemic reasons such as the patient's coagulation mechanism disorder, $\mathrm{PB}$ is closely related to the surgeon's operating skills, experience, and seriousness (9). Bleeding can be divided into the following types. (I) Bleeding in the space between the skin flap and the anterior cervical muscle: It is manifested by the apparent bulging of the neck incision after surgery. The patient feels no pressure and has no symptoms of dyspnea. However, some patients may experience neck discomfort and swelling pain. If the condition is not discovered quickly, ecchymoses appear on the skin and spread to the surroundings. The most common causes are bleeding from the anterior jugular vein, bleeding in the infrahyoid muscles and the sternocleidomastoid muscle, and bleeding from the subcutaneous tissue. (II) Bleeding in the thyroid bed: In case of bleeding in this space, the patient immediately feels neck pressure, obvious swelling pain in the front of the neck, emotional tension, restlessness, difficulty breathing, and sometimes typical depression of the suprasternal fossa, the supraclavicular fossa, and the intercostal spaces while breathing or may even suffocate or die. The common causes include arterial bleeding, venous bleeding, thyroid stump bleeding, bleeding in the infrahyoid muscles, blood oozing from the surgical wound, coagulation dysfunction, and other systemic factors. To prevent bleeding after thyroid surgery, a surgeon should the following: (i) avoid massive ligation of thyroid-innervating arteries and veins and ligate blood vessels separately after ossification; (ii) avoid cutting a large number of tissues concurrently using energy devices and ensure that each cut is closed; (iii) avoid electrocoagulation of small arterial branches and use ligation; (iv) carefully stop 
bleeding on muscle surfaces; (v) handle the thyroid stump appropriately to avoid invalid suturing and falling sutures; (vi) routinely use warm normal saline to wash the wound, and relieve the spasmodic blood vessels so that bleeding points can be identified easily; (vii) the surgical site was wiped with gauze, the blood or indefinite thrombosis was removed, and active bleeding was confirmed; (viii) ask the anesthesiologist to increase the intrathoracic pressure (Valsalva maneuver) or make the patient's head droop $30^{\circ}$, which helps to find venous bleeding; (ix) assess the coagulation function of the patient before surgery and actively treat the patient symptomatically if his/her coagulation function is poor (48); (x) Hemostatic agents can be used before closing the strap or platysma muscle layers, but the use of hemostatic agents in thyroid surgery yields minimal advantages for the management of perioperative bleeding risk $(49,50)$.

After thyroid surgery, the patient's blood pressure, pulse, oxygen saturation, and breathing should be monitored closely. Once PB is detected, a quick judgment should be made on whether conservative treatment or surgical treatment is required. (I) Conservative treatment: if the drainage is unobstructed, the fluid in the drain is not bright red and is a small volume, the patient feels no local pain and does not have difficulty breathing, and his/her oxygen saturation, blood pressure, and pulse are stable, he/she may be placed under observation. Because bleeding in the space between the skin flap and the anterior cervical muscle has little effect on the respiratory tracts, and bleeding in this space is usually blood oozing from the wound or bleeding from small veins, conservative treatment may be effective; however, close observation is required. Once the patient experiences difficulty breathing or swelling in the front of the neck worsens, immediate surgical treatment is required. (II) Surgical treatment: whether immediate removal of the sutures on-bed and opening the incision to relieve the airway compression and whether tracheal intubation or onbed tracheotomy is required should be judged appropriately. After providing decisive and accurate treatment, the surgeon should operate again to examine the incision. When removing clots during surgery, the surgeon should focus on the areas where clots are concentrated and the areas whose surrounding muscle tissues turn dark; there may be bleeding points near these areas. After removing the clots, the surgeon should wash the wound with warm water and look for the bleeding points as there could be more than one bleeding point. Random clamping should be avoided during surgery, as it may cause injury to vital structures such as the RLN. After locating the bleeding points and controlling the bleeding, the surgeon should wash the wound with warm normal saline to reduce the risk of infection. Complying to the principle of aseptic operation, antibacterial drugs should be administered after the surgery as necessary (51).

\section{Right lymphatic duct or thoracic duct injury}

Right lymphatic duct or thoracic duct injury induces lymphatic leakage and chylous leakage, with the incidence in simple thyroid surgery as $0.5-1.4 \%$ and that in neck dissection as $2-8 \%$ (52). The risk of lymphatic leak is based on lymph node dissection. The risk of lymphatic leak is much higher in lateral neck dissection compared to central neck dissection. Additionally, as the left side poses much higher risk due to the location of the thoracic duct. Simple lymphatic leakage has little effect on patients' whole-body condition, while massive chylous leakage causes blood electrolyte disorder, decreased blood volume, local infection, mediastinal infection, and flap necrosis in patients, leading to neck macrovascular hemorrhage and be life-threatening.

The key to preventing the right lymphatic duct or thoracic duct injury is a mastery of their respective anatomical structure. When the lymph nodes of the superior clavicle and the inferior segment of the internal jugular vein are removed during surgery, the probability of injury is increased by avoiding to dissect the lymphatic duct; nonetheless, a delicate operation is required to prevent tearing of the wall of the lymphatic duct. The right lymphatic duct or thoracic duct mostly injects into multiple trunks, and hence, proper clamping and ligation are required at each step. Moreover, if a transparent and clear liquid flows out intraoperatively, the leak should be found, ligated, and closed by suturing together with the surrounding tissue. Subsequently, medicalbiological gels are sprayed when the suture and ligation of the lymphatic duct leak are not satisfactory. Importantly, energy equipment is not recommended in this area, and even if used, ligation should be supplemented. At the end of the surgery, the patient's chest and abdomen should be pressed, or the anesthesiologist should be asked to increase intrathoracic pressure (Valsalva maneuver) to check for any clear fluid outflow from the surgical site. In the event of postoperative lymphatic or chylous leakage, conservative treatment is performed in most cases (53). (I) Conservation treatment: (i) dietary control and nutritional support; (ii) continuous suction and pressure dressing; (iii) when the accumulation of chylous fluid leads to inflammation, and the compression of the flap increases the risk of infection, 
or there is an intra-mediastinal infection in severe cases, appropriate antibiotic treatment is recommended. Surgical treatment should be performed as early as possible in the event of failed conservative treatment. (II) Surgical treatment: the leaks should be searched intraoperatively while avoiding new injury. Ligation should be applied to the leak(s), which often cannot be sutured and ligated together with the surrounding tissue because the latter is edematous and brittle. At this point, the surgery can be transferred to the adjacent muscle flap, which can be stuffed and reinforced by spraying biological gels sprayed in the space (54).

\section{Tracheomalacia and tracheal collapse}

Asphyxia occurs in the event of tracheomalacia and tracheal collapse, which is associated with the surgeon's misjudgment of the disease. In the cases with long medical history and huge goiter, preoperative examinations aid in making a risk judgment of tracheomalacia and tracheal collapse. For patients with huge goiter, prolonged compression of the trachea leads to poor blood circulation in the tracheal wall and degenerative changes, thinning, weakened elasticity, and atrophy of the cricoid tracheal cartilage. Concurrently, tracheal respiration is maintained with the support of the thyroid and the surrounding tissue (55). After the thyroid tissue is surgically removed, the trachea loses dependence and support, and the softened trachea collapses, causing airway obstruction. If the number of the softened cricoid cartilage is $<3$, the tracheal suspension is performed intraoperatively (56), wherein the trachea is suspended anterolaterally to both sides of the tracheal wall; otherwise, tracheotomy is conducted. When it is difficult to judge whether the trachea is softened intraoperatively, tracheotomy is recommended to ensure smooth airway in the patients (57).

\section{Laryngeal edema}

According to the literature, the incidence of laryngeal edema after thyroid surgery is about 3\% (58). Asphyxia caused by laryngeal edema is fatal. The common causes are as follows: (I) tracheal intubation is not smooth during anesthesia, and repeated operations lead to laryngeal contusion and edema; (II) hemorrhage and hematoma lead to disrupted laryngeal venous return, further causing laryngeal edema; (III) prolonged lymphatic leakage or venous bleeding leads to laryngeal edema; (IV) prolonged surgical time and repeated operations near the larynx stimulate the organ and cause laryngeal edema; (V) patients with concurrent respiratory tract infections exhibit postoperative symptoms of increased secretion and recurrent cough and undergo laryngeal edema and viscous sputum blockage, which induces asphyxia. The preventive measures are as follows: (I) for patients with difficult intubation, a video-assisted laryngoscopy operation by an experienced anesthesiologist is recommended; (II) close postoperative observation to detect hemorrhage and lymphatic leakage early is essential to provide treatment promptly, which reduces the occurrence of laryngeal edema; (III) when the surgery is predicted to be prolonged, dexamethasone should be administered intraoperatively to reduce stress response; (IV) after surgery, active measures, such as inhalation of nebulized medicine for eliminating phlegm, are adopted to control respiratory tract infection and antibiotics to control infection, if necessary. In the event of laryngeal edema, steroid hormones and bronchodilators are administered to reduce stress response; also, symptomatic treatment is required (58). However, if the effects are not satisfactory, tracheotomy is recommended.

\section{Tracheospasm and laryngospasm}

Main tracheospasm refers to the pathological phenomenon wherein patients undergo dyspnea, asphyxia, and also die because of increased tracheal resistance and impaired ventilation due to tracheal smooth muscle contraction and narrowing of the tracheal cavity (59). Intraoperative hypoxia and intraoperative tracheal stimulation (repeated physical stimulation of the trachea and thermal energy of electric knife and other equipment during surgery) might induce main tracheospasm; postoperative extubation and irritable cough are also triggering factors. Most of the patients with tracheospasm have a history of airway hyperresponsiveness, asthma, and smoking. As a result, the preoperative prediction of possible tracheospasm is the key to prevention. (I) Dexamethasone should be administered intraoperatively to reduce stress response; (II) surgical operations should be gentle to avoid overstimulation of the trachea; (III) sharp excision is recommended at the time of separating the thyroid from the trachea; (IV) postoperative nebulized inhalation lubricates the airway to reduce its hyperresponsiveness and avoid irritable cough. Emergency tracheal intubation is performed in the event of tracheospasm, and tracheotomy is required when necessary (60). Similarly, laryngospasm after thyroidectomy could also result to dyspnea, and asphyxia. The reasons are often related to the hypocalcemia and vocal cord paralysis $(61,62)$. 
Some of the reasons are the same to the tracheospasm. In case of urgency, the patients were treated generally with CPAP ventilation, re-intubation, deepen the anesthesia, paralyze with succinylcholine (intramuscular or intralingual or intraosseous), and give atropine $0.02 \mathrm{mg} / \mathrm{kg}$, etc (63). Main branch of ansa cervicalis nerve to RLN anastomosis could be efficient management for paroxysmal laryngospasm due to RLN injury (62).

\section{Tracheal or esophageal injury}

Tracheal or esophageal injury is a rare complication, and intraoperative esophageal injury is even rarer due to the anatomical position of the esophagus (64). Tracheal or esophageal injury caused by endoscopic surgery or energy equipment might be difficult to detect during surgery, leading to severe postoperative consequences. Postoperative patients with tracheal injury exhibit subcutaneous emphysema, which is worsened after cough, possibly followed by mediastinal emphysema. In the case of patients with complicated malignant tumor surgery or surgical history, the evaluation of the correlation among lesion and trachea should be based on the results of the preoperative imaging examination. Then, preparations are made for intraoperative tracheotomy. When a partial trachea is removed to reconstruct the airway during surgery, the suture line should run through the whole layer of the trachea. Also, knot tightness needs to be consistent to prevent postoperative tracheal leakage. After suturing, saline is injected into the surgical site, and the anesthesiologist pressurizes the airway to observe for air leakage (64). The treatment of some rare cases, such as tracheal cyst, is based on the clinical experience of surgeons. In the event of postoperative tracheal leakage, if the air leakage is small, most patients recover by reinforced suction and pressure dressing. Otherwise, the tracheal leak is repaired surgically and tracheotomy.

Postoperative patients with esophageal injury exhibit turbid drainage or food outflow after eating, accompanied by local swelling and pain. In the case of patients with complicated malignant tumor surgery or surgical history, the evaluation of the correlation among lesion and esophagus should be based on the results of the preoperative imaging examination. Then, preparations are made for preoperative gastric tube indwelling to judge the course of the esophagus during surgery. If the tumor does not invade the esophageal mucosa, the esophageal myometrium is repaired directly; however, if the tumor invades the entire layer of the esophagus, a portion of the esophagus is removed, and the flap sutured and repaired. Furthermore, postoperative esophageal fistula is a severe complication, and high esophageal fistula cannot heal easily. Patients are recommended long-term fasting and water deprivation, intragastric or enteral nutrition or intravenous nutrition, adequate drainage, and anti-inflammatory symptomatic treatment. If conservative treatment fails, surgical repair is conducted; a part of the esophagus is excised, the flap repaired, or the esophagus is replaced with a tubular stomach (65).

\section{Incision scar formation}

Moderate scar formation after thyroid surgery is a normal response of the body to repair trauma, but a hypertrophic scar caused by excessive scar formation is a pathological manifestation, which is a frequent occurrence in the incisions of open thyroid surgery, especially in the "L"shaped incisions of neck dissection. The hypertrophic scars bring psychological and physical pain to patients with a severe impact on their social activities and neck functions (66). People with yellow skin have thick dermal tissue and high collagen content promote the occurrence of scar formation in those with white skin. Moreover, scar formation is also more likely to occur in young individuals than in the elderly because the former have high levels of hormones and developed sebaceous glands (67). Such individual cases are beyond control, and hence, the treatment of incision during and after surgery is very critical. Neck incision is class I aseptic incision, but infection, tissue necrosis, sutures, and other foreign body residues may result in inflammation. The excessive release of inflammatory cytokines leads to delayed epidermal regeneration, increased granulation tissue, and scar formation. Similarly, during the intraoperative incision of the skin and subcutaneous tissue, no sharp incision is fashioned, or the skin is scalded by an electric knife, causing inflammatory responses and excessive scar formation. Mechanical tension plays a critical role in neck scar formation, which is the result of excessive tension, excessive proliferation of skin fibroblasts, and increased collagen synthesis (68). Tranilast and silica gel polymer are used during the early postoperative period to prevent hypertrophic scars (69). For scars already formed, Triamcinolone Acetonide or Diprospan is injected locally or physiotherapy, such as intense pulsed light laser, ultrapulse $\mathrm{CO}_{2}$ laser, and the ion beam are recommended $(70,71)$. Taken together, early prevention is better than treatment 
for patients with a history of scar formation.

\section{Skin sinus formation}

Skin sinus formation is one of the rare complications after thyroid surgery, with an incidence of about $0.08 \%$ (72). Patients exhibit the following symptoms: when the suture of the incision is removed and the incision heals after thyroid surgery, there are discomfort and pain in the surgical site, ulceration, and suppuration in the neck skin. However, after a dressing change, the infection is controlled, and the incision heals gradually. But ulceration and suppuration recur, following which the infected area shows scarring, and then the sinus tract is formed, with or without general malaise and fever. Intriguingly, sinus formation is closely associated with the surgical method and foreign body residues in the thyroid fossa (72). The principle of surgery is to eliminate the etiology, remove the foreign body, clear the infected area, and resect the sinus tract.

\section{Conclusions}

Thyroid surgery has become a routine and safe procedure of operation with a decline in the overall morbidity and mortality due to complications. However, operative complications might contribute to negative impact on quality of life after surgery. Therefore, it is the responsibility of every thyroid surgeon to remain alert and aware of the occurrence of various intraoperative and postoperative complications and exercise effective prevention and treatment. In addition, many complications that effect patients are much better tolerated if the patient has appropriate expectations of what the complications are and how to treat them. If patients are more aware of the complications going into surgery, they have less anxiety when they occur because they knew what to expect and are aware of the possible treatments. Open communication between surgeon and patient optimizes the potential negative effects that complications may have on patients' quality of life.

\section{Acknowledgments}

Funding: This work is supported by the Inner Mongolia Autonomous Region "prairie outstanding ability person" project.

\section{Footnote}

Reporting Checklist: The authors have completed the Narrative review reporting checklist. Available at http:// dx.doi.org/10.21037/gs-20-859

Conflicts of Interest: Both authors have completed the ICMJE uniform disclosure form (available at http://dx.doi. org/10.21037/gs-20-859). SJ reports grants from Inner Mongolia Autonomous Region "prairie outstanding ability person" project, during the conduct of the study. IS has no conflicts of interest to declare.

Ethical Statement: The authors are accountable for all aspects of the work in ensuring that questions related to the accuracy or integrity of any part of the work are appropriately investigated and resolved.

Open Access Statement: This is an Open Access article distributed in accordance with the Creative Commons Attribution-NonCommercial-NoDerivs 4.0 International License (CC BY-NC-ND 4.0), which permits the noncommercial replication and distribution of the article with the strict proviso that no changes or edits are made and the original work is properly cited (including links to both the formal publication through the relevant DOI and the license). See: https://creativecommons.org/licenses/by-nc-nd/4.0/.

\section{References}

1. Sarkar S, Banerjee S, Sarkar R, et al. A Review on the History of 'Thyroid Surgery'. Indian J Surg 2016;78:32-6.

2. Hannan SA. The magnificent seven: a history of modern thyroid surgery. Int J Surg 2006;4:187-91.

3. Bobanga ID, McHenry CR. Treatment of patients with Graves' disease and the appropriate extent of thyroidectomy. Best Pract Res Clin Endocrinol Metab 2019;33:101319.

4. Rosato L, Avenia N, Bernante P, et al. Complications of thyroid surgery: analysis of a multicentric study on 14,934 patients operated on in Italy over 5 years. World J Surg 2004:28:271-6.

5. Duclos A, Peix JL, Colin C, et al. Influence of experience on performance of individual surgeons in thyroid surgery: prospective cross sectional multicentre study. BMJ 2012;344:d8041. 
6. Nicholson KJ, Teng CY, McCoy KL, et al. Completion thyroidectomy: A risky undertaking? Am J Surg 2019;218:695-9.

7. Caulley L, Johnson-Obaseki S, Luo L, et al. Risk factors for postoperative complications in total thyroidectomy: A retrospective, risk-adjusted analysis from the National Surgical Quality Improvement Program. Medicine (Baltimore) 2017;96:e5752.

8. Christou N, Mathonnet M. Complications after total thyroidectomy. J Visc Surg 2013;150:249-56.

9. Chahardahmasumi E, Salehidoost R, Amini M, et al. Assessment of the Early and Late Complication after Thyroidectomy. Adv Biomed Res 2019;8:14.

10. Scerrino G, Melfa G, Raspanti C, et al. Minimally Invasive Video-Assisted Thyroidectomy: Analysis of Complications From a Systematic Review. Surg Innov 2019;26:381-7.

11. Păduraru DN, Ion D, Carsote M, et al. Postthyroidectomy Hypocalcemia - Risk Factors and Management. Chirurgia (Bucur) 2019;114:564-70.

12. Caglià P, Puglisi S, Buffone A, et al. Post-thyroidectomy hypoparathyroidism, what should we keep in mind?. Ann Ital Chir 2017;6:371-81.

13. Bai B, Chen Z, Chen W. Risk factors and outcomes of incidental parathyroidectomy in thyroidectomy: A systematic review and meta-analysis. PLoS One 2018;13:e0207088.

14. Mohebati A, Shaha AR. Anatomy of thyroid and parathyroid glands and neurovascular relations. Clin Anat 2012;25:19-31.

15. Ladurner R, Lerchenberger $\mathrm{M}, \mathrm{Al}$ Arabi N, et al. Parathyroid Autofluorescence-How Does It Affect Parathyroid and Thyroid Surgery? A 5 Year Experience. Molecules 2019;24:2560.

16. Abbaci M, De Leeuw F, Breuskin I, et al. Parathyroid gland management using optical technologies during thyroidectomy or parathyroidectomy: A systematic review. Oral Oncol 2018;87:186-96.

17. Kim BW, Kim SY, Lee YS, et al. Parathyroid score can predict the duration of required calcium supplementation after total thyroidectomy. PLoS One 2017;12:e174088.

18. Lorente-Poch L, Sancho JJ, Ruiz S, et al. Importance of in situ preservation of parathyroid glands during total thyroidectomy. Br J Surg 2015;102:359-67.

19. Sitges-Serra A, Lorente-Poch L, Sancho J. Parathyroid autotransplantation in thyroid surgery. Langenbecks Arch Surg 2018;403:309-15.

20. Tartaglia F, Blasi S, Giuliani A, et al. Parathyroid autotransplantation during total thyroidectomy. Results of a retrospective study. Int J Surg 2016;28 Suppl 1:S79-83.

21. Hillary SL, Guillermet S, Brown NJ, et al. Use of methylene blue and near-infrared fluorescence in thyroid and parathyroid surgery. Langenbecks Arch Surg 2018;403:111-8.

22. Ladurner R, Al Arabi N, Guendogar U, et al. Nearinfrared autofluorescence imaging to detect parathyroid glands in thyroid surgery. Ann R Coll Surg Engl 2018;100:33-6.

23. Kose E, Rudin AV, Kahramangil B, et al. Autofluorescence imaging of parathyroid glands: An assessment of potential indications. Surgery 2020;167:173-9.

24. Huang SM. Do we overtreat post-thyroidectomy hypocalcemia?. World J Surg 2012;36:1503-8.

25. Dedivitis RA, Aires FT, Cernea CR. Hypoparathyroidism after thyroidectomy: prevention, assessment and management. Curr Opin Otolaryngol Head Neck Surg 2017;25:142-6.

26. Shah M, Bancos I, Thompson GB, et al. Teriparatide Therapy and Reduced Postoperative Hospitalization for Postsurgical Hypoparathyroidism. JAMA Otolaryngol Head Neck Surg 2015;141:822-7.

27. Palermo A, Mangiameli G, Tabacco G, et al. PTH(134) for the Primary Prevention of Postthyroidectomy Hypocalcemia: The THYPOS Trial. J Clin Endocrinol Metab 2016;101:4039-45.

28. Hayward NJ, Grodski S, Yeung M, et al. Recurrent laryngeal nerve injury in thyroid surgery: a review. ANZ J Surg 2013;83:15-21.

29. Gambardella C, Polistena A, Sanguinetti A, et al. Unintentional recurrent laryngeal nerve injuries following thyroidectomy: Is it the surgeon who pays the bill?. Int J Surg 2017;41:S55-9.

30. Kaisha W, Wobenjo A, Saidi H. Topography of the recurrent laryngeal nerve in relation to the thyroid artery, Zuckerkandl tubercle, and Berry ligament in Kenyans. Clin Anat 2011;24:853-7.

31. Henry BM, Pękala PA, Sanna B, et al. The Anastomoses of the Recurrent Laryngeal Nerve in the Larynx: A MetaAnalysis and Systematic Review. J Voice 2017;31:495-503.

32. Benkhadoura M, Taktuk S, Alobedi R. Recurrent laryngeal nerve injury and hypoparathyroidism rates in reoperative thyroid surgery. Turk J Surg 2017;33:14-7.

33. Bakalinis E, Makris I, Demesticha T, et al. Non-Recurrent Laryngeal Nerve and Concurrent Vascular Variants: A Review. Acta Med Acad 2018;47:186-92.

34. Ramouz A, Rasihashemi SZ, Safaeiyan A, et al. Comparing postoperative complication of LigaSure 
Small Jaw instrument with clamp and tie method in thyroidectomy patients: a randomized controlled trial (IRCT2014010516077N1). World J Surg Oncol 2018;16:154.

35. Pisanu A, Porceddu G, Podda M, et al. Systematic review with meta-analysis of studies comparing intraoperative neuromonitoring of recurrent laryngeal nerves versus visualization alone during thyroidectomy. J Surg Res 2014;188:152-61.

36. Higgins TS, Gupta R, Ketcham AS, et al. Recurrent laryngeal nerve monitoring versus identification alone on post-thyroidectomy true vocal fold palsy: a meta-analysis. Laryngoscope 2011;121:1009-17.

37. Lyu Z, Xu W, Zou J, et al. Recurrent laryngeal nerve decompression for bilateral recurrent laryngeal nerve paralyses after thyroid surgery. Zhonghua Er Bi Yan Hou Tou Jing Wai Ke Za Zhi 2014;49:885-8.

38. Hong JW, Roh TS, Yoo HS, et al. Outcome with immediate direct anastomosis of recurrent laryngeal nerves injured during thyroidectomy. Laryngoscope 2014;124:1402-8.

39. Yumoto E, Sanuki T, Kumai Y. Immediate recurrent laryngeal nerve reconstruction and vocal outcome. Laryngoscope 2006;116:1657-61.

40. Miyauchi A, Inoue H, Tomoda C, et al. Improvement in phonation after reconstruction of the recurrent laryngeal nerve in patients with thyroid cancer invading the nerve. Surgery 2009;146:1056-62.

41. Potenza AS, Araujo Filho VJF, Cernea CR. Injury of the external branch of the superior laryngeal nerve in thyroid surgery. Gland Surg 2017;6:552-62.

42. Ortega C, Maranillo E, McHanwell S, et al. External laryngeal nerve landmarks revisited. Head Neck 2018;40:1926-33.

43. Cheruiyot I, Kipkorir V, Henry BM, et al. Surgical anatomy of the external branch of the superior laryngeal nerve: a systematic review and meta-analysis. Langenbecks Arch Surg 2018;403:811-23.

44. Naytah M, Ibrahim I, da Silva S. Importance of incorporating intraoperative neuromonitoring of the external branch of the superior laryngeal nerve in thyroidectomy: A review and meta-analysis study. Head Neck 2019;41:2034-41.

45. Wojtczak B, Aporowicz M, Kaliszewski K, et al. Consequences of bleeding after thyroid surgery - analysis of 7805 operations performed in a single center. Arch Med Sci 2018;14:329-35.

46. Liu J, Sun W, Dong W, et al. Risk factors for post- thyroidectomy haemorrhage: a meta-analysis. Eur J Endocrinol 2017;176:591-602.

47. Su L, Li J, Tang X, et al. Therapeutic Effects of Bipolar Coagulation Forceps on Open Thyroid Surgery. Rev Invest Clin 2016;68:256-61.

48. Lee HS, Lee BJ, Kim SW, et al. Patterns of Postthyroidectomy Hemorrhage. Clin Exp Otorhinolaryngol 2009;2:72-7.

49. Materazzi G, Ambrosini CE, Fregoli L, et al. Prevention and management of bleeding in thyroid surgery. Gland Surg 2017;6:510-5.

50. Khadra H, Bakeer M, Hauch A, et al. Hemostatic agent use in thyroid surgery: a meta-analysis. Gland Surg 2018;7:S34-41.

51. Reinhart HA, Snyder SK, Stafford SV, et al. Same day discharge after thyroidectomy is safe and effective. Surgery 2018;164:887-94.

52. Delaney SW, Shi H, Shokrani A, et al. Management of Chyle Leak after Head and Neck Surgery: Review of Current Treatment Strategies. Int J Otolaryngol 2017;2017:8362874.

53. Polistena A, Vannucci J, Monacelli M, et al. Thoracic duct lesions in thyroid surgery: An update on diagnosis, treatment and prevention based on a cohort study. Int J Surg 2016;28 Suppl 1:S33-7.

54. Park I, Her N, Choe JH, et al. Management of chyle leakage after thyroidectomy, cervical lymph node dissection, in patients with thyroid cancer. Head Neck 2018;40:7-15.

55. Agarwal A, Mishra AK, Gupta SK, et al. High incidence of tracheomalacia in longstanding goiters: experience from an endemic goiter region. World J Surg 2007;31:832-7.

56. Jin S, Bao W. Huge thyroid adenoma with tracheomalacia. Clin Case Rep 2018;6:1635-6.

57. Findlay JM, Sadler GP, Bridge H, et al. Postthyroidectomy tracheomalacia: minimal risk despite significant tracheal compression. Br J Anaesth 2011;106:903-6.

58. Sanjeeva KK, Chandra B, Balakrishna MA, et al. ClinicoEpidemiological Study and Treatment Outcome of Multinodular Goitre at A Tertiary Care Hospital. J Clin Diagn Res 2015;9:PC22-5.

59. Bourdeaux C, Benton J. Post intubation airway obstruction in thyroid surgery. Anaesthesia 2003;58:187-8.

60. Yildirim E. Principles of Urgent Management of Acute Airway Obstruction. Thorac Surg Clin 2018;28:415-28.

61. Kakava K, Tournis S, Papadakis G, et al. Postsurgical Hypoparathyroidism: A Systematic Review. In Vivo 
2016;30:171-9.

62. Wang W, Sun J, Tang H, et al. Main branch of ACN-toRLN for management of laryngospasm due to unilateral vocal cord paralysis. Laryngoscope 2020;130:2412-9.

63. Tellan G, Marandola M, Fiengo L, et al. Acute pulmonary oedema associated with laryngospasm after thyroidectomy: a case report. Chir Ital 2008;60:303-9.

64. Tartaglia N, Iadarola R, Di Lascia A, et al. What is the treatment of tracheal lesions associated with traditional thyroidectomy? Case report and systematic review. World J Emerg Surg 2018;13:15.

65. Li Z, Liu CP, Shi L, et al. Operation for differentiated thyroid cancer: a experience of 546 cases. Zhonghua Wai Ke Za Zhi 2008;46:375-7.

66. Choi Y, Lee JH, Kim YH, et al. Impact of postthyroidectomy scar on the quality of life of thyroid cancer patients. Ann Dermatol. 2014;26:693-9.

67. Kim HI, Kwak CY, Kim HY, et al. Correlation between dermal thickness and scar formation in female patients

Cite this article as: Jin S, Sugitani I. Narrative review of management of thyroid surgery complications. Gland Surg 2021;10(3):1135-1146. doi: 10.21037/gs-20-859 after thyroidectomy. Arch Craniofac Surg 2018;19:120-6.

68. Shin JU, Park JH, Oh SH, et al. Early intervention in thyroidectomy scars: demographics, symptoms, and prevention. J Wound Care 2015;24:163-4, 166-8, 170-1.

69. Kohavi L, Sprecher E, Zur E, et al. The Effect of Tranilast 8\% Liposomal Gel Versus Placebo on Post-Cesarean Surgical Scars: A Prospective Double-Blind Split-Scar Study. Dermatol Surg 2017;43:1157-63.

70. Yun JS, Choi YJ, Kim WS, et al. Prevention of thyroidectomy scars in Asian adults using a 532-nm potassium titanyl phosphate laser. Dermatol Surg 2011;37:1747-53.

71. Park YJ, Kim SJ, Song HS, et al. Prevention of Thyroidectomy Scars in Asian Adults With Low-Level Light Therapy. Dermatol Surg 2016;42:526-34.

72. Jin S, Bao W, Borkhuu O, et al. Clinical Study on the Etiology of Postthyroidectomy Skin Sinus Formation. Surg Res Pract 2017;2017:5283792. 